PHYSICAL REVIEW D 91, 104038 (2015)

\title{
Acoustic clouds: Standing sound waves around a black hole analogue
}

\author{
Carolina L. Benone* and Luís C. B. Crispino ${ }^{\dagger}$ \\ Faculdade de Física, Universidade Federal do Pará, 66075-110 Belém, Pará, Brazil \\ Carlos Herdeiro \\ Departamento de Física da Universidade de Aveiro and CIDMA, Campus de Santiago, \\ 3810-183 Aveiro, Portugal \\ (Received 29 January 2015; published 29 May 2015)
}

\begin{abstract}
Under certain conditions sound waves in fluids experience an acoustic horizon with analogue properties to those of a black hole event horizon. In particular, a draining bathtub-like model can give rise to a rotating acoustic horizon and hence a rotating black hole (acoustic) analogue. We show that sound waves, when enclosed in a cylindrical cavity, can form stationary waves around such rotating acoustic holes. These acoustic perturbations display similar properties to the scalar clouds that have been studied around Kerr and Kerr-Newman black holes; thus they are dubbed acoustic clouds. We make the comparison between scalar clouds around Kerr black holes and acoustic clouds around the draining bathtub explicit by studying also the properties of scalar clouds around Kerr black holes enclosed in a cavity. Acoustic clouds suggest the possibility of testing, experimentally, the existence and properties of black hole clouds, using analog models.
\end{abstract}

DOI: 10.1103/PhysRevD.91.104038

PACS numbers: 04.50.-h, 04.20.Jb, 04.50.Kd

\section{INTRODUCTION}

In a renowned work, Unruh showed that sound waves in an irrotational flow of an inviscid and barotropic fluid are governed by the Klein-Gordon equation on an effective geometry, called effective acoustic spacetime [1]. He observed, moreover, that the effective geometry can be analogous to a black hole $(\mathrm{BH})$ spacetime: If the fluid flow becomes supersonic, the surface separating subsonic and supersonic flows is perceived as a one-way membrane by sound waves. Indeed, this surface is an event horizon of the effective acoustic spacetime.

Building on Unruh's work, a variety of analogue models has been developed (see, e.g., [2,3]) with the promise of constructing $\mathrm{BH}$ analogues in the laboratory that could be used to test the physical properties of BHs. It is therefore of interest to understand if a given property observed in $\mathrm{BH}$ spacetimes can be mimicked by analogue models. Within this context, $\mathrm{BH}$ properties like absorption [4,5], scattering [6,7], quasinormal modes, and Regge poles [8-12] have been investigated in acoustic analogue spacetimes over the past few years.

Another such BH property, which has been the subject of recent studies, is the existence of stationary bound states of a massive scalar field, $\Phi$, around Kerr (or Kerr-Newman) BHs [13-17]: scalar clouds. ${ }^{1}$ These clouds exist for

\footnotetext{
*lben.carol@gmail.com

†crispino@ufpa.br

therdeiro@ua.pt

\$eugen.radu@ua.pt

${ }^{1}$ See also [18,19] for a marginal charged scalar and Proca clouds around Reissner-Nordström BHs.
}

monochromatic modes with frequency $\omega$ and azimuthal harmonic index $m$, when the condition

$$
\omega=\omega_{c} \equiv m \Omega_{H}
$$

holds, where $\Omega_{H}$ is the horizon angular velocity. ${ }^{2}$ Consequently, these clouds are interpreted as zero modes of the superradiant instability of Kerr BHs, which amplifies modes obeying $\omega<m \Omega_{H}$ [20]. Alternatively, one may regard this condition as requiring that a field mode of type $\Phi \sim e^{-i \omega t} e^{i m \phi}$, using standard Boyer-Lindquist coordinates, is preserved by the horizon null generator $k=\partial_{t}+\Omega_{H} \partial_{\phi}$. Physically, this guarantees the absence of scalar field flux through the horizon, a necessary requirement for the existence of stationary bound states around the $\mathrm{BH}$.

Besides being of interest on their own, as equilibrium states of a matter field around a $\mathrm{BH}$, scalar clouds have been related to the existence of Kerr BHs with scalar hair [15] (see also [21,22]): Backreacting clouds generate a new family of solutions to the Einstein-(massive)-Klein-Gordon system, continuously connected to the Kerr family. This suggests that whenever clouds of a given matter field can be found around $\mathrm{a} \mathrm{BH}$, in a linear analysis and with a stationary and axisymmetric energy-momentum tensor, there exists a fully nonlinear solution of new hairy BHs corresponding to making these clouds heavy and thus deforming the geometry [23]. A more fundamental

\footnotetext{
${ }^{2}$ In the Kerr-Newman case, for a charged scalar field with charge $q$, this condition is modified to $\omega=m \Omega_{H}+q \Phi_{H}$, where $\Phi_{H}$ is the horizon electrostatic potential.
} 
mechanism for the existence of hairy BHs, however, that does not require the existence of clouds in a linear analysis but does require the zero flux condition $\omega=m \Omega_{H}$ to hold, may be at place, as illustrated by the examples in [24,25].

In this paper we will show that there are cloudlike configurations around $\mathrm{BH}$ analogue models. Specifically, we shall be interested in acoustic holes and a $[(2+1)$ dimensional] draining bathtub model for which the acoustic horizon is rotating [26]. Acoustic perturbations of this model are described by a massless Klein-Gordon equation, unlike the aforementioned scalar clouds which occur for a massive Klein-Gordon field. The role of the mass is to provide the necessary confinement for bound states to exist. In a realistic experiment with a draining bathtub, the "spacetime" cannot extend forever, and will be enclosed in a cavity, which we assume to be cylindrical as to respect the isometries of the background. Such a cavity implements a boundary condition that replaces the effect of the mass and, when the analogue of condition (1) holds, produces standing wavelike bound states, which we call acoustic clouds.

To make the comparison between these acoustic clouds and scalar clouds around a Kerr BH sharper we shall also study scalar clouds of the massless Klein-Gordon equation for a Kerr BH enclosed in a cavity. Here the cavity is spheroidal ( $r=$ const) in Boyer-Lindquist coordinates, and two different boundary conditions for the scalar field are implemented therein. We will show that the variation of the clouds' properties with the mirror location and "quantum numbers" labeling the cloud is qualitatively similar for both acoustic and scalar clouds. Let us remark that superradiance of scalar fields around BHs in a cavity has been studied in the past, for both Kerr [20,27,28] and Reissner-Nordström geometries [29-31].

This paper is organized as follows. In Sec. II we analyze massless scalar clouds around Kerr BHs in a cavity. We will see that their properties are reminiscent of the massive case (without mirror). This sets a reference frame for addressing the acoustic clouds in a draining bathtub model, which is done in Sec. III. In both Secs. II and III, computations are done using numerical methods. We shall briefly comment on the (un)likelihood of describing acoustic clouds analytically. We close in Sec. IV with some final remarks.

We assume throughout the paper $\hbar=G=1$.

\section{SCALAR CLOUDS AROUND KERR BHS}

\section{A. Background and scalar equation}

Let us first consider the Kerr background, described by the line element

$$
\begin{aligned}
d s^{2}= & -\frac{\Delta}{\rho^{2}}\left(d t-a \sin ^{2} \theta d \phi\right)^{2}+\frac{\rho^{2}}{\Delta} d r^{2} \\
& +\rho^{2} d \theta^{2}+\frac{\sin ^{2} \theta}{\rho^{2}}\left[\left(r^{2}+a^{2}\right) d \phi-a d t\right]^{2},
\end{aligned}
$$

with

$$
\rho^{2} \equiv r^{2}+a^{2} \cos ^{2} \theta, \quad \Delta \equiv r^{2}-2 M r+a^{2} .
$$

$M$ is the Arnowitt-Deser-Misner (ADM) mass and $J=a M$ is the ADM angular momentum of the $\mathrm{BH}$. We consider in this section $c=1$.

To solve the Klein-Gordon equation for a massless scalar field, $\square \Phi=0$, on this background, we make a harmonic/ Fourier decomposition of the scalar field, which is therefore considered to be a sum of modes of the type $\Phi_{l m}=e^{i(m \phi-\omega t)} S_{l m}(\theta) R_{l m}(r)$. Taking $S_{l m}$ to be spheroidal harmonics, obeying

$$
\begin{aligned}
& \frac{1}{\sin \theta} \frac{d}{d \theta}\left(\sin \theta \frac{d S_{l m}}{d \theta}\right) \\
& \quad+\left(K_{l m}-a^{2} \omega^{2}+a^{2} \omega^{2} \cos ^{2} \theta-\frac{m^{2}}{\sin ^{2} \theta}\right) S_{l m}=0
\end{aligned}
$$

where $K_{l m}$ are separation constants, the above decomposition of the scalar field permits a full separation of variables. The radial functions $R_{l m}(r)$ obey the radial equation

$$
\Delta \frac{d}{d r}\left(\Delta \frac{d R_{l m}}{d r}\right)+\left[H^{2}+\left(2 m a \omega-K_{l m}\right) \Delta\right] R_{l m}=0,
$$

where $H \equiv\left(r^{2}+a^{2}\right) \omega-a m$. We can rewrite Eq. (5) using the tortoise coordinates, defined by

$$
\frac{d r_{*}}{d r} \equiv \frac{r^{2}+a^{2}}{\Delta},
$$

and obtain a new radial equation without the first derivative term,

$$
\begin{aligned}
& \frac{d^{2} U_{l m}}{d r_{*}^{2}}+\left\{\frac{\left[H^{2}+\left(2 m a \omega-K_{l m}\right) \Delta\right]}{\left(r^{2}+a^{2}\right)^{2}}\right. \\
& \left.-\frac{\Delta(\Delta+2 r(r-M))}{\left(r^{2}+a^{2}\right)^{3}}+\frac{3 r^{2} \Delta^{2}}{\left(r^{2}+a^{2}\right)^{4}}\right\} U_{l m}=0,
\end{aligned}
$$

with

$$
U_{l m} \equiv R_{l m} \sqrt{r^{2}+a^{2}} .
$$

We are now able to analyze the behavior of the radial solution close and far away from the BH. In the limit $r \rightarrow$ $r_{H}$ we require a purely ingoing wave, while in the asymptotic case we have an outgoing wave:

$$
R_{l m}(r) \approx \begin{cases}e^{-i\left(\omega-\omega_{c}\right) r_{*}}, & \text { for } r \rightarrow r_{H}, \\ e^{i \omega r_{*}}, & \text { for } r \rightarrow \infty .\end{cases}
$$


With the solutions given by (9) no stationary solutions exist. To have such solutions we shall impose, besides $\omega=\omega_{c}$, a different boundary condition, as we shall now describe.

\section{B. Numerical procedure and results}

To find clouds, i.e., bound statelike solutions, we enclose the system in a cavity whose boundary is located at

$$
r=r_{0} .
$$

We impose two different boundary conditions to our problem: The first is of a Dirichlet type such that the scalar field vanishes at the cavity's boundary, $R_{l m}\left(r_{0}\right)=0$. The second is a Neumann boundary condition such that the derivative of the field vanishes at the cavity's boundary, $d R_{l m}\left(r_{0}\right) / d r=0$. These, together with condition (1), allow us to find massless scalar clouds around Kerr BHs. The clouds are labeled by three "quantum" numbers: $l$, the spheroidal harmonic index, $m$ the azimuthal harmonic index, and $n$, the node number of the radial function.

To obtain the radial function, we shall integrate Eq. (5) numerically. For that purpose, we consider the following series expansions for the separation constants $K_{l m}$ :

$$
K_{l m}-a^{2} \omega^{2}=l(l+1)+\sum_{k=1}^{\infty} c_{k} a^{2 k} \omega^{2 k},
$$

where the coefficients $c_{k}$ are given in [32], and to initiate the radial integration we take the following series expansion:

$$
R_{l m}=R_{0}\left(1+\sum_{k \geq 1} R_{k}\left(r-r_{H}\right)^{k}\right)
$$

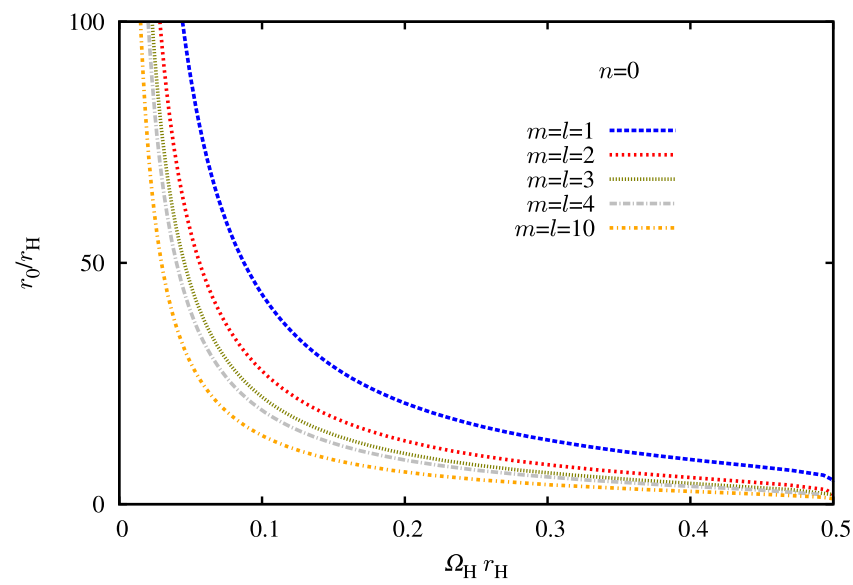

close to the horizon. To find the coefficients $R_{k}$ we substitute Eq. (12) in Eq. (5) and expand it in terms of $\left(r-r_{H}\right)$, taking $R_{0}=1$. We end up with a shooting problem for the radial function, using the shooting parameter $a$. Fixing the quantum numbers $(n, l$, and $m)$ and using the event horizon $r_{H}$ as a scaling factor, we choose values for $r_{0}$, solve the radial equations from very close to the event horizon to the position of the mirror, and find the values of $a$ that obey the appropriate boundary condition.

In Fig. 1 we display the mirror location $r_{0}$ in terms of the horizon angular velocity $\Omega_{H}$, both scaled to the horizon radius, keeping $m=l$ and varying the cloud quantum number $m$, for both Dirichlet boundary conditions (left panel) and Neumann boundary conditions (right panel). The first observation is that, generically, $r_{0} / r_{H} \rightarrow \infty$, $\Omega_{H} \rightarrow 0$. This illustrates the fact that there are no massless clouds for Schwarzschild BHs in a cavity. Indeed this is expected from the known results of the massive case: There are no (massive) scalar clouds for the Schwarzschild BH, even though it is possible to have arbitrarily long-lived quasibound states [33]. The second generic observation is that, as we approach the mirror to the BH horizon we need to increase $\Omega_{H}$ in order to have clouds. Conversely, and taking precisely the extremal case $\left(\Omega_{H} r_{H}=0.5\right)$, we observe that increasing the value of $m=l$ the position of the mirror tends to the horizon. We shall come back to this point shortly.

The dependence on the cloud's quantum numbers observed in Fig. 1 can be summarized as follows. Increasing $l=m$, implies that, for fixed mirror position $r_{0} / r_{H}$, clouds exist for smaller horizon angular velocity $\Omega_{H} r_{H}$. This trend is analogous to the one observed for massive scalar clouds (without mirror) in [17]. They can be heuristically interpreted in terms of a mechanical equilibrium between the $\mathrm{BH}$-cloud gravitational attraction and

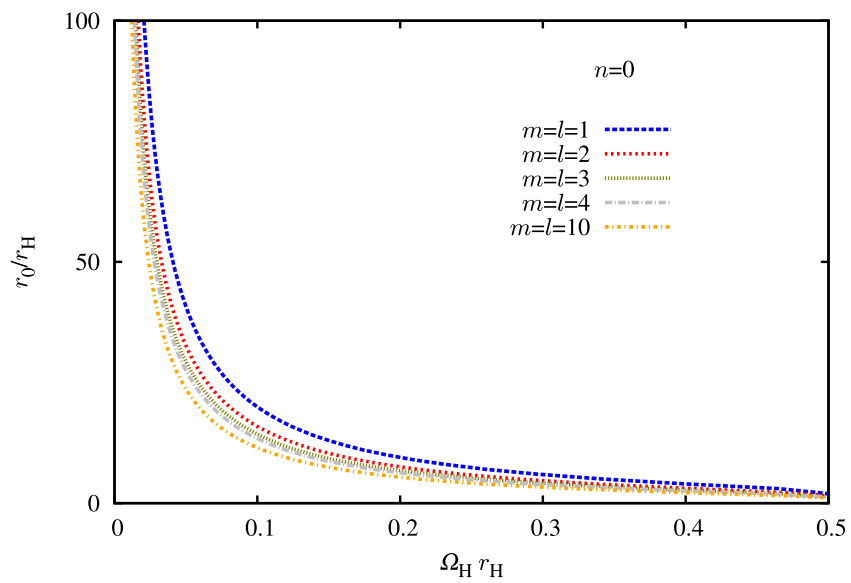

FIG. 1 (color online). Massless scalar clouds around Kerr BHs surrounded by a mirror at $r=r_{0}$ with a Dirichlet (left panel) or a Neumann (right panel) boundary condition. In both panels we keep $n=0, m=l$ and vary the cloud's quantum number $m$, as specified in the key. 

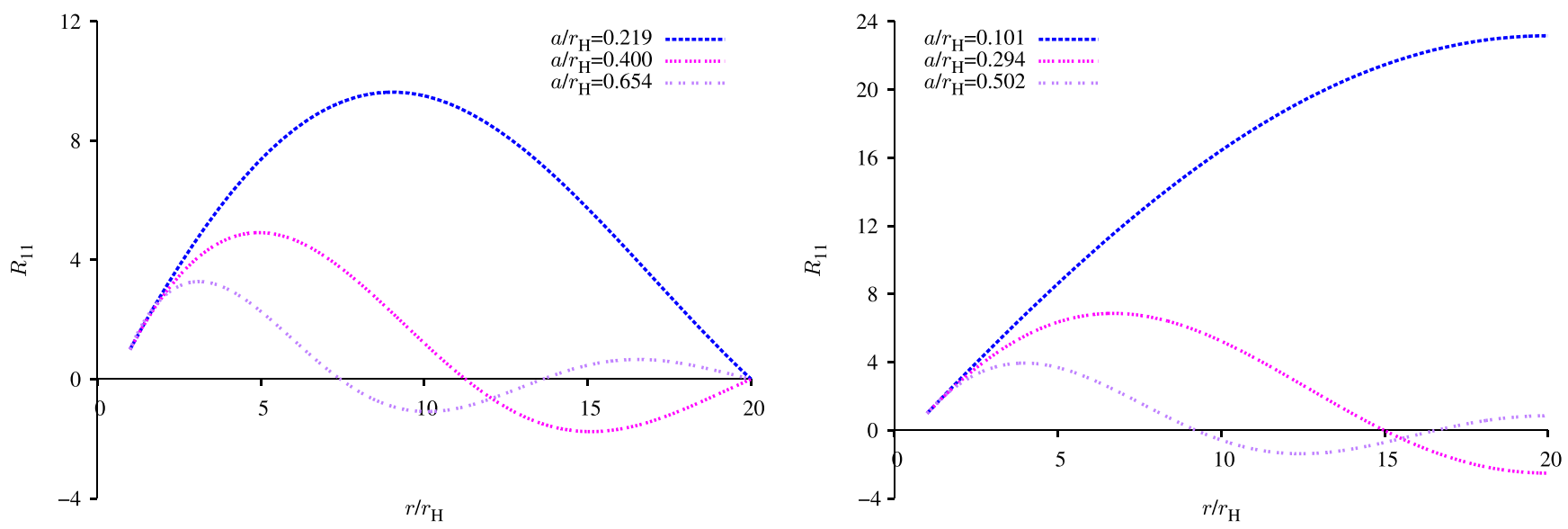

FIG. 2 (color online). Radial solutions $R_{11}$ with $r_{0} / r_{H}=20$ for Dirichlet (left panel) and Neumann (right panel) boundary conditions.

angular momentum driven repulsion due to the BH-cloud energy currents, cf. [17]. ${ }^{3}$

Finally, concerning the differences between Dirichlet and Neumann boundary conditions, Fig. 1 shows that both cases present the same qualitative behavior, but that for the same angular velocity $\Omega_{H}$, clouds with Neumann boundary conditions occur for a smaller $r_{0}$. This is quite natural, as one can always obtain a Neumann cloud from a Dirichlet cloud by decreasing the cavity where the latter stands to the point where the radial derivative of the radial function vanishes, which occurs inside the cavity.

Figure 2 illustrates the radial profile of the massless scalar clouds for Dirichlet (left panel) and Neumann (right panels) boundary conditions for $l=m=1$. For both cases, we choose $r_{0}=20 r_{H}$ and show three distinct solutions corresponding to different numbers of nodes $n=0,1$, and 2 . The radial profile has the typical form of standing waves with fixed boundary conditions.

Let us comment that we have verified qualitatively similar clouds exist not only for massive but also for self-interacting scalar fields around Kerr BHs in cavity, generalizing the ones studied in [25] for asymptotically flat spacetimes.

\section{ACOUSTIC CLOUDS IN A DRAINING BATHTUB}

\section{A. Background and acoustic perturbations}

We now turn to the acoustic $\mathrm{BH}$ analogue obtained from the draining bathtub model considered in [26]. The scalar field now describes acoustic perturbations on a fluid flow. We still consider it to be a complex scalar field, but all the results apply for a real scalar field as well, simply by taking

\footnotetext{
${ }^{3}$ We would also like to comment that increasing either the spheroidal harmonic index $l$ or the node number $n$, implies that, for fixed mirror position $r_{0} / r_{H}$, clouds exist for larger horizon angular velocity $\Omega_{H} r_{H}$, as also happens in the mirrorless massive case.
}

the real (or imaginary) part of the result. The effective acoustic geometry corresponds to the metric seen by sound waves traveling in a fluid with flow velocity given by

$$
\vec{v}=\frac{A}{r} \hat{r}+\frac{B}{r} \hat{\phi},
$$

where the constants $A$ and $B$ are the draining and the circulation, respectively. For this model the following line element is obtained:

$$
\begin{aligned}
d s^{2}= & -\left(1-\frac{A^{2}+B^{2}}{r^{2}}\right) d t^{2}+\left(1-\frac{A^{2}}{r^{2}}\right)^{-1} d r^{2} \\
& -2 B d \phi d t+r^{2} d \phi^{2} .
\end{aligned}
$$

Thus, the effective geometry is $1+2$ dimensional. It has an event horizon at $r_{H}=A$; this horizon is rotating with angular velocity $\Omega_{H}=B / A^{2}$. We consider in this section $c_{S}=1$, where $c_{S}$ is the speed of sound.

In order to find the solution of the Klein-Gordon equation we again consider a Fourier/mode decomposition. The modes have the form

$$
\Phi_{m}(r, \phi, t)=e^{i(m \phi-\omega t)} \psi_{m}(r),
$$

where $\psi_{m}(r)$ obeys

$$
\begin{aligned}
& \frac{1}{r}\left(1-\frac{A^{2}}{r^{2}}\right) \frac{d}{d r}\left[r\left(1-\frac{A^{2}}{r^{2}}\right) \frac{d \psi_{m}}{d r}\right] \\
& \quad+\left[\omega^{2}-\frac{2 B m \omega}{r^{2}}-\frac{m^{2}}{r^{2}}\left(1-\frac{A^{2}+B^{2}}{r^{2}}\right)\right] \psi_{m}=0
\end{aligned}
$$

We can rewrite Eq. (16) as

$$
\frac{d^{2}}{d r_{*}^{2}} \zeta_{m}+\left[\left(\omega-\frac{B m}{r^{2}}\right)^{2}-V_{m}(r)\right] \zeta_{m}=0
$$

with 

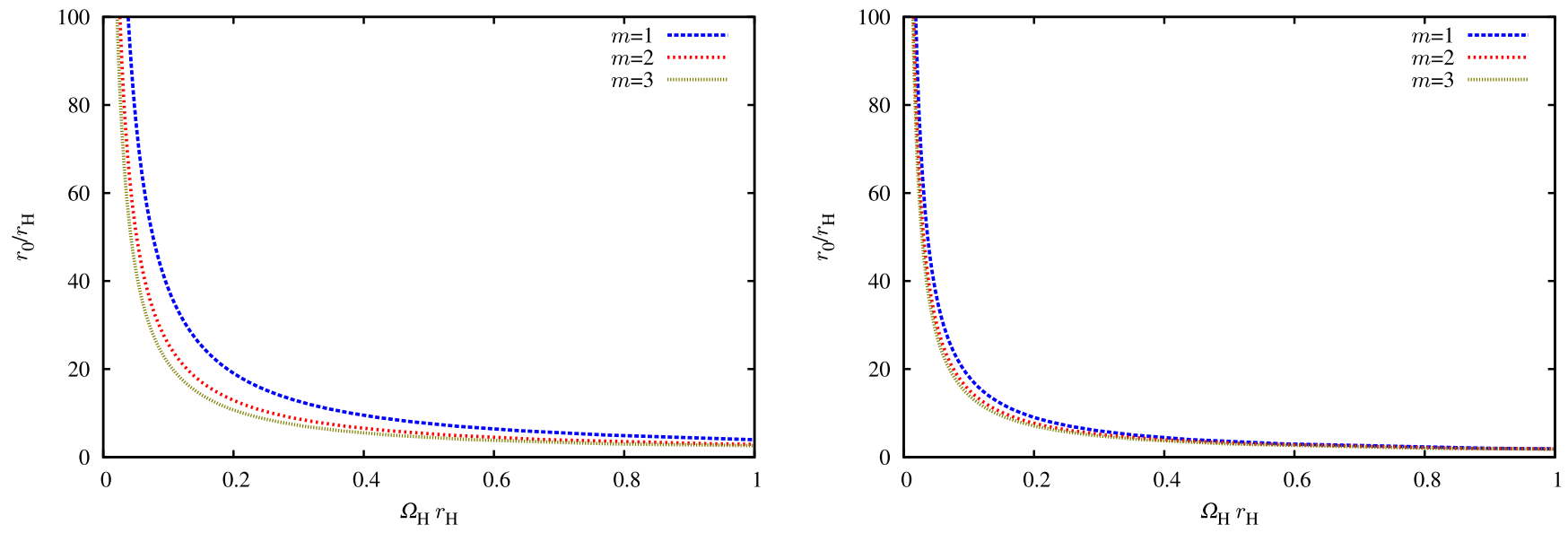

FIG. 3 (color online). Acoustic clouds on the draining bathtub surrounded by a mirror at $r=r_{0}$ for Dirichlet (left panel) boundary conditions and Neumann (right panel) boundary conditions. In these panels we keep $m=1$ and vary the cloud quantum number $n$, as specified in the key.
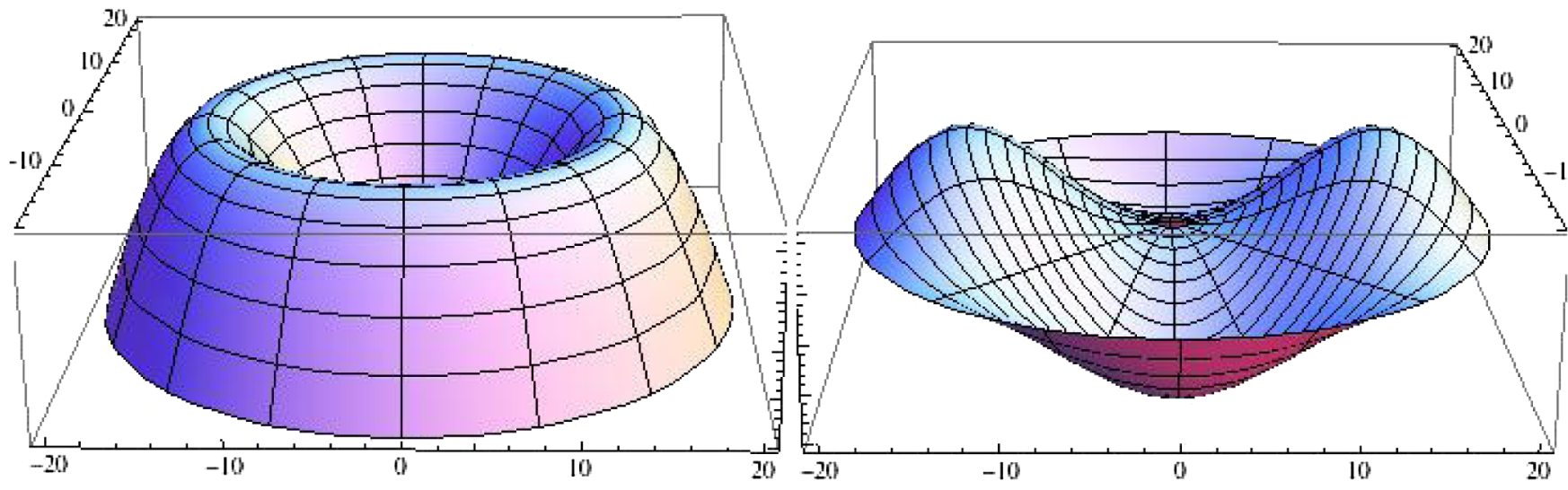

FIG. 4 (color online). Spatial distribution of nodeless acoustic clouds with Dirichlet boundary condition and $m=2$, on the $r, \phi$ plane. On the left panel we present $\left|\Phi_{m}\right|$, while on the right panel we show the real part of $\Phi_{m}$ for a fixed $t$. In both plots the outer edge is the cylindrical mirror and the inner boundary is the acoustic BH horizon. We have fixed $r_{0} / r_{H}=20$ and the cloud supporting background has $B / r_{H} \simeq 0.13$.

$$
V_{m}(r)=\left(1-\frac{A^{2}}{r^{2}}\right)\left[\frac{m^{2}-1 / 4}{r^{2}}+\frac{5 A^{2}}{4 r^{2}}\right]
$$

where $\zeta_{m}=\sqrt{r} \psi_{m}$ and the Regge-Wheeler coordinate can be defined by

$$
\frac{d}{d r_{*}}=\left(1-\frac{A^{2}}{r^{2}}\right) \frac{d}{d r}
$$

We find that the solutions at the horizon and at infinity behave similarly as before

$$
\psi_{\omega m}(r) \approx \begin{cases}e^{-i\left(\omega-\omega_{c}\right) r_{*}}, & \text { for } r \rightarrow r_{H}, \\ e^{i \omega r_{*},}, & \text { for } r \rightarrow \infty,\end{cases}
$$

with

$$
\omega_{c}=m B / A^{2}=m \Omega_{H} .
$$

\section{B. Numerical procedure and results}

Superradiant scattering of acoustic waves can be seen in this type of geometries, without the need of introducing a cavity $[4,8,34]$. To obtain acoustic clouds, however, we need to consider $\omega=\omega_{c}$ and introduce a mirror at $r=r_{0}$ where boundary conditions of Dirichlet $\left[\psi_{m}\left(r_{0}\right)=0\right]$ or Neumann $\left[d\left(\psi_{m}(r)\right) / d r\right]$ types are imposed. ${ }^{4}$ In the present case the clouds will be labeled by only two quantum numbers: the azimuthal harmonic index $m$ and the node number $n$.

The numerical procedure is analogous to that adopted in Sec. II B. We consider the power series expansion

\footnotetext{
${ }^{4}$ These boundary conditions are associated to a cylindrical mirror with low (Dirichlet) or high (Neumann) acoustic impedance $[35,36]$, the latter being perhaps more easily implemented experimentally, since it corresponds to a rigid boundary cylinder.
} 

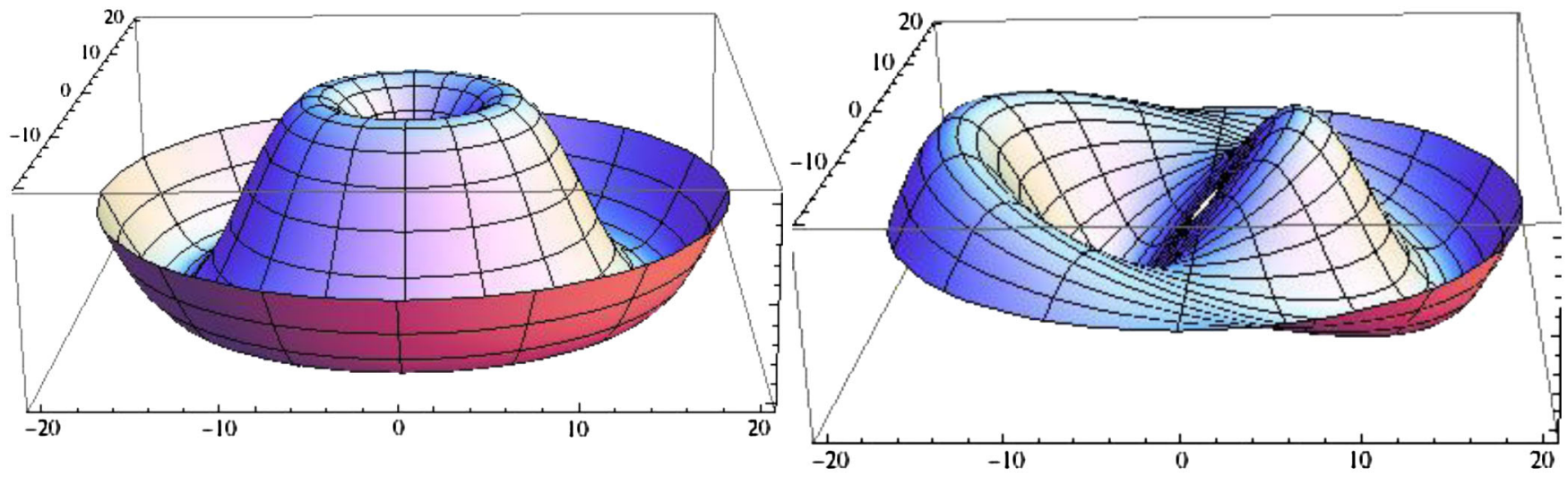

FIG. 5 (color online). Same as in Fig. 4 but for nodeful acoustic clouds with $n=1$ and $m=1$, on the $r, \phi$ plane. Again, we have fixed $r_{0} / r_{H}=20$ and the cloud supporting background has $B / r_{H} \simeq 0.35$.
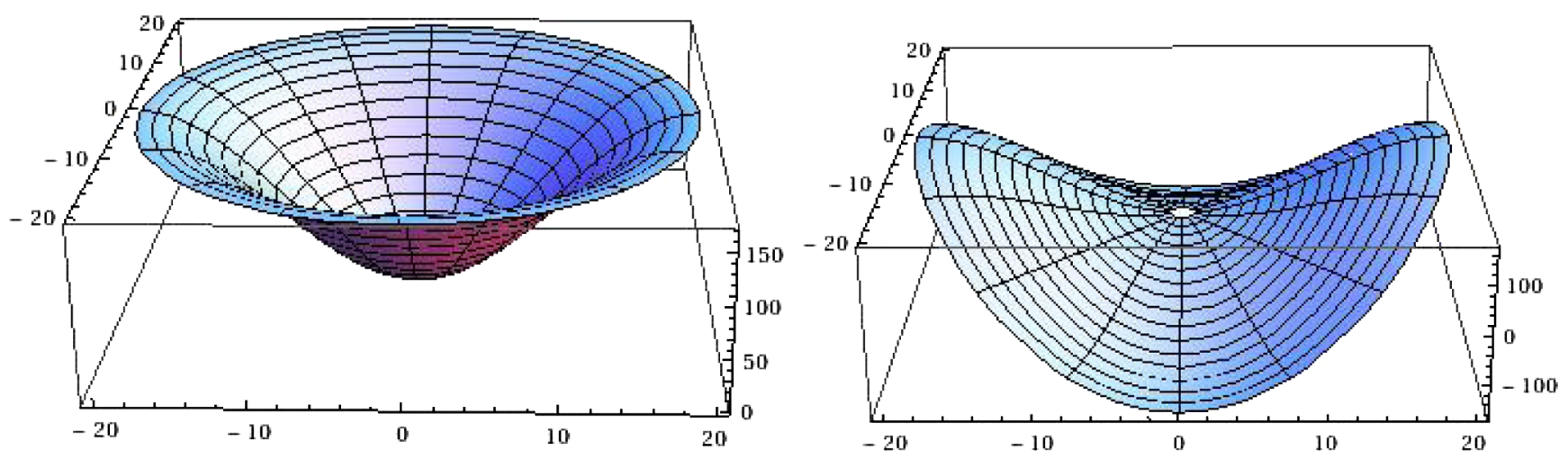

FIG. 6 (color online). Same as in Fig. 4 but for Neumann boundary conditions. Again, we have fixed $r_{0} / r_{H}=20$ and the cloud supporting background has $B / r_{H} \simeq 0.08$.

$$
\psi_{m}=\psi_{0}\left(1+\sum_{k \geq 1} \psi_{k}\left(r-r_{H}\right)^{k}\right)
$$

close to the horizon. To find the coefficients $\psi_{k}$ we substitute Eq. (22) in Eq. (16) and expand it in terms of $\left(r-r_{H}\right)$, taking $\psi_{0}=1$. We end up with a shooting problem for the radial function with shooting parameter $B$. Fixing the quantum numbers $(n$ and $m$ ) and using the event horizon $r_{H}=A$ as a scaling factor, we choose values for $r_{0}$, solve the radial equations from close to the event horizon until the position of the mirror, and find the values of $B$ that obey the appropriate boundary condition.
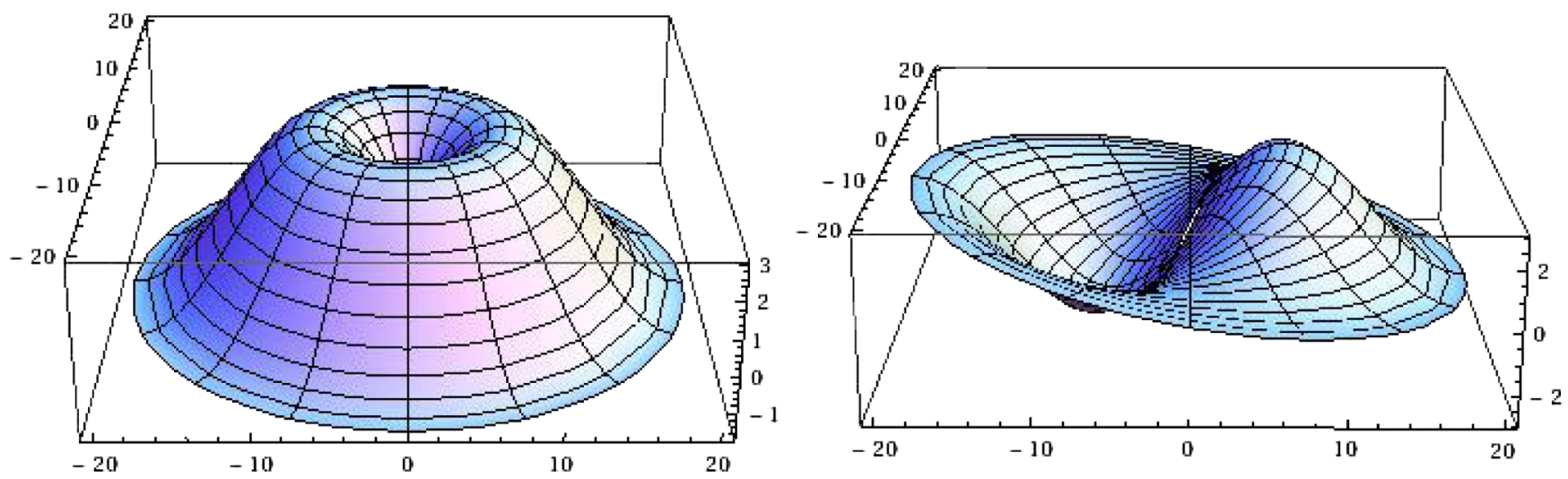

FIG. 7 (color online). Same as in Fig. 5 but for Neumann boundary conditions (i.e., nodeful acoustic clouds with $n=1$ and $m=1$ on the $r, \phi$ plane). Again, we have fixed $r_{0} / r_{H}=20$ and the cloud supporting background has $B / r_{H} \simeq 0.27$. 
In Fig. 3 we compare clouds with different quantum numbers for Dirichlet and Neumann boundary conditions. The observed behavior is completely analogous to that seen for the Kerr case discussed above: (i) as $r_{0} / r_{H} \rightarrow \infty$, $\Omega_{H} \rightarrow 0$, (ii) as we approach the mirror to the acoustic hole $\Omega_{H}$ increases, (iii) as $m$ decreases, the angular velocity $\Omega_{H} r_{H}$ increases for fixed mirror position $r_{0} / r_{H}$, and (iv) for the Neumann boundary condition, the existence lines occur for smaller values of $\Omega_{H}$, for the same $r_{0}{ }^{5}$

In Figs. 4 and 5 we exhibit 3D plots with the radial and azimuthal profiles of some examples of acoustic clouds for the Dirichlet boundary condition, without and with nodes, respectively. In Figs. 6 and 7 we exhibit the same as in Figs. 4 and 5, but for the Neumann boundary condition.

One may wonder if an analytic solution may be found for Eq. (16). Studies of the draining bathtub model have actually concluded that the radial function in this case can be expressed in terms of Heun functions (see, e.g., [37]). It seems, however, that not much use can be made of these functions to find the relevant information to obtain the acoustic clouds. Moreover, for the acoustic hole, there is no theoretical restriction that prevents taking $B>A$; in other words, there is no extremal case for this analogue model, for which there could be some extra simplification for the radial equation, as it happens for the clouds around extremal Kerr BHs [13].

\section{CONCLUSION}

The connection between the perturbations of a Newtonian fluid flow and the relativistic physics described by a curved metric - the acoustic spacetime - is a surprising and remarkable one. This connection between what seems to be a priori two very different worlds can be useful both ways: to use the technology of Lorentzian geometry to learn about hydrodynamics and to use (experimentally controllable) fluids to learn about general relativity and, in particular, BH physics. In this paper we have been concerned about the former viewpoint and have added yet another property seen in $\mathrm{BH}$ physics to the list that can be mimicked by a $\mathrm{BH}$ acoustic analogue: the existence of standing waves of a scalar field around a rotating $\mathrm{BH}$; these take the form of acoustic clouds around a $\mathrm{BH}$ analogue.

\footnotetext{
${ }^{5}$ As for the previous case, as we increase $n$ the angular velocity $\Omega_{H} r_{H}$ increases for fixed mirror position $r_{0} / r_{H}$.
}

One of the insights that may be obtained from studying analogue models is to separate phenomena that are intrinsic to general relativity from those that occur whenever Lorentzian geometry may be involved. The example we have provided shows that the existence of stationary waves of a scalar field in the presence of an event horizon is not unique to the BHs of general relativity and suggests they occur for a large class of $\mathrm{BH}$ analogues described by an appropriate Lorentzian geometry, regardless of the dynamical equations obeyed by the background. On the other hand, the equations that rule the background are crucial to promote the scalar clouds seen around Kerr BHs to scalar hairy BHs [15]. This is the point at which the parallelism between the BHs from general relativity and its acoustic analogues is likely to end: Whereas for the former Kerr BHs with scalar hair exist, continuously connecting to the Kerr BHs that support scalar clouds, it is unclear if any notion of a deformed acoustic hole by backreacting acoustic clouds can be defined.

Finally, it is an exciting possibility that the acoustic clouds described herein could be seen in a laboratory experiment. Experimental research on analogue systems is ongoing, but still in its infancy (see [38] for a list of references). We hope the result described here can provide a further research direction for these experiments.

\section{ACKNOWLEDGMENTS}

We would like to thank Juan Carlos Degollado for comments on a draft of this paper. The work in this paper is supported by the FCT Investigator program, Grants No. PTDC/FIS/116625/2010, No. NRHEP295189-FP7-PEOPLE-2011-IRSES, and the CIDMA strategic funding UID/MAT/04106/2013. The authors would like also to thank Conselho Nacional de Desenvolvimento Científico e Tecnológico (CNPq), Coordenação de Aperfeiçoamento de Pessoal de Nível Superior (CAPES), and Fundação Amazônia Paraense de Amparo à Pesquisa (FAPESPA), from Brazil, for partial financial support.

Note added.-After the completion of this work, S. Hod posted a paper where the Kerr system with a mirror is studied [39]. The main focus therein is the minimum mirror radius which allows the existence of clouds. The general trend observed in [39] agrees with our results, whereas the (semianalytic) technique used therein applies only to the special case of fast spinning Kerr BHs. 
[1] W. Unruh, Experimental black hole evaporation, Phys. Rev. Lett. 46, 1351 (1981).

[2] C. Barcelo, S. Liberati, and M. Visser, Analogue gravity, Living Rev. Relativity 8, 12 (2005).

[3] Analogue Spacetimes: The First Thirty Years, edited by V. Cardoso, L. C. B. Crispino, S. Liberati, E. S. Oliveira, and M. Visser (Editora Livraria da Física, São Paulo, 2013).

[4] L. C. B. Crispino, E. S. Oliveira, and G. E. A. Matsas, Absorption cross section of canonical acoustic holes, Phys. Rev. D 76, 107502 (2007).

[5] E. S. Oliveira, S. R. Dolan, and L. C. B. Crispino, Absorption of planar waves in a draining bathtub, Phys. Rev. D 81, 124013 (2010).

[6] S. R. Dolan, E. S. Oliveira, and L. C. B. Crispino, Scattering of sound waves by a canonical acoustic hole, Phys. Rev. D 79, 064014 (2009).

[7] S. R. Dolan and E. S. Oliveira, Scattering by a draining bathtub vortex, Phys. Rev. D 87, 124038 (2013).

[8] E. Berti, V. Cardoso, and J. P. S. Lemos, Quasinormal modes and classical wave propagation in analogue black holes, Phys. Rev. D 70, 124006 (2004).

[9] V. Cardoso, J. P. S. Lemos, and S. Yoshida, Quasinormal modes and stability of the rotating acoustic black hole: Numerical analysis, Phys. Rev. D 70, 124032 (2004).

[10] S. R. Dolan, L. A. Oliveira, and L. C. B. Crispino, Quasinormal modes and Regge poles of the canonical acoustic hole, Phys. Rev. D 82, 084037 (2010).

[11] S. R. Dolan, L. A. Oliveira, and L. C. B. Crispino, Resonances of a rotating black hole analogue, Phys. Rev. D 85, 044031 (2012).

[12] J. P. S. Lemos, Rotating analogue black holes: Quasinormal modes and tails, superresonance, and sonic bombs and plants in the draining bathtub acoustic hole, arXiv:1312.7176.

[13] S. Hod, Stationary scalar clouds around rotating black holes, Phys. Rev. D 86, 104026 (2012).

[14] S. Hod, Stationary resonances of rapidly-rotating Kerr black holes, Eur. Phys. J. C 73, 2378 (2013).

[15] C. A. R. Herdeiro and E. Radu, Kerr Black Holes with Scalar Hair, Phys. Rev. Lett. 112, 221101 (2014).

[16] S. Hod, Kerr-Newman black holes with stationary charged scalar clouds, Phys. Rev. D 90, 024051 (2014).

[17] C. L. Benone, L. C. B. Crispino, C. Herdeiro, and E. Radu, Kerr-Newman scalar clouds, Phys. Rev. D 90, 104024 (2014).

[18] J. C. Degollado and C. A. Herdeiro, Stationary scalar configurations around extremal charged black holes, Gen. Relativ. Gravit. 45, 2483 (2013).

[19] M. O. P. Sampaio, C. Herdeiro, and M. Wang, Marginal scalar and Proca clouds around Reissner-Nordström black holes, Phys. Rev. D 90, 064004 (2014).

[20] W. H. Press and S. A. Teukolsky, Floating Orbits, Superradiant Scattering and the Black-hole Bomb, Nature (London) 238, 211 (1972).
[21] C. Herdeiro and E. Radu, Ergosurfaces for Kerr black holes with scalar hair, Phys. Rev. D 89, 124018 (2014).

[22] C. Herdeiro and E. Radu, Construction and physical properties of Kerr black holes with scalar hair, arXiv:1501.04319.

[23] C. A. R. Herdeiro and E. Radu, A new spin on black hole hair, Int. J. Mod. Phys. D 23, 1442014 (2014).

[24] Y. Brihaye, C. Herdeiro, and E. Radu, Myers-Perry black holes with scalar hair and a mass gap, Phys. Lett. B 739, 1 (2014).

[25] C. Herdeiro, E. Radu, and H. Runarsson, Non-linear Qclouds around Kerr black holes, Phys. Lett. B 739, 302 (2014).

[26] M. Visser, Acoustic black holes: horizons, ergospheres and Hawking radiation, Classical Quantum Gravity 15, 1767 (1998).

[27] V. Cardoso, O. J. C. Dias, J. P. S. Lemos, and S. Yoshida, Black-hole bomb and superradiant instabilities, Phys. Rev. D 70, 044039 (2004).

[28] S. R. Dolan, Superradiant instabilities of rotating black holes in the time domain, Phys. Rev. D 87, 124026 (2013).

[29] J. C. Degollado, C. A. R. Herdeiro, and H. F. Rúnarsson, Rapid growth of superradiant instabilities for charged black holes in a cavity, Phys. Rev. D 88, 063003 (2013).

[30] S. Hod, Analytic treatment of the charged black-hole-mirror bomb in the highly explosive regime, Phys. Rev. D 88, 064055 (2013).

[31] J. C. Degollado and C. A. R. Herdeiro, Time evolution of superradiant instabilities for charged black holes in a cavity, Phys. Rev. D 89, 063005 (2014).

[32] M. Abramowitz and I. A. Stegun, Handbook of Mathematical Functions with Formulas, Graphs, and Mathematical Tables (Dover, New York, 1964).

[33] J. Barranco, A. Bernal, J. C. Degollado, A. Diez-Tejedor, M. Megevand, M. Alcubierre, D. Núñez, and O. Sarbach, Schwarzschild Black Holes can Wear Scalar Wigs, Phys. Rev. Lett. 109, 081102 (2012).

[34] S. Basak and P. Majumdar, 'Superresonance' from a rotating acoustic black hole, Classical Quantum Gravity 20, 3907 (2003).

[35] M. Lax and H. Feshbach, Absorption and Scattering for Impedance Boundary Conditions on Spheres and Circular Cylinders, J. Acoust. Soc. Am. 20, 108 (1948).

[36] L. A. Oliveira, V. Cardoso, and L. C. B. Crispino, Ergoregion instability: The hydrodynamic vortex, Phys. Rev. D 89, 124008 (2014).

[37] H. Vieira and V. Bezerra, Scalar exact solutions and phononic Hawking radiation in acoustic black holes, arXiv:1406.6884.

[38] M. Richartz, A. Prain, S. Liberati, and S. Weinfurtner, Rotating black holes in a draining bathtub: superradiant scattering of gravity waves, arXiv:1411.1662.

[39] S. Hod, Onset of superradiant instabilities in the composed Kerr-black-hole-mirror bomb, Phys. Lett. B 736, 398 (2014). 